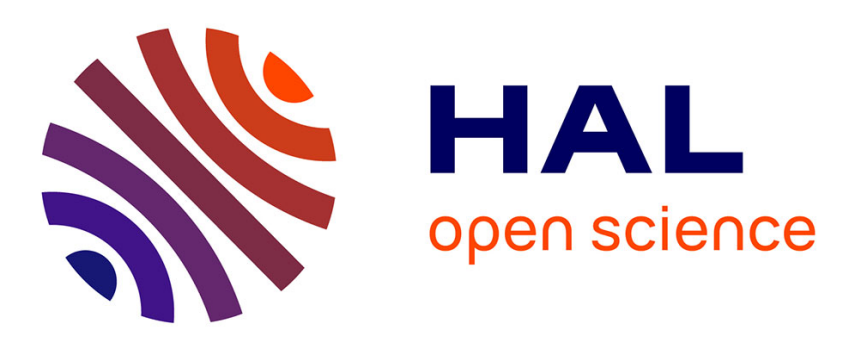

\title{
La transmission des valeurs sociales et familiales dans l'océan Indien: Présentation du dossier
}

Thierry Malbert, Gérard Pithon

\section{To cite this version:}

Thierry Malbert, Gérard Pithon. La transmission des valeurs sociales et familiales dans l'océan Indien : Présentation du dossier. Revue internationale de l'éducation familiale, 2015, Familles et éducation dans l'océan Indien, 38, pp.11-25. 10.3917/rief.038.0011 . hal-01336584

\section{HAL Id: hal-01336584 \\ https://hal.univ-reunion.fr/hal-01336584}

Submitted on 30 Jun 2016

HAL is a multi-disciplinary open access archive for the deposit and dissemination of scientific research documents, whether they are published or not. The documents may come from teaching and research institutions in France or abroad, or from public or private research centers.
L'archive ouverte pluridisciplinaire $\mathbf{H A L}$, est destinée au dépôt et à la diffusion de documents scientifiques de niveau recherche, publiés ou non, émanant des établissements d'enseignement et de recherche français ou étrangers, des laboratoires publics ou privés. 


\title{
La transmission des valeurs sociales et familiales dans l'océan Indien
}

\author{
Présentation du dossier
}

\author{
Thierry Malbert et Gérard Pithon ${ }^{1}$
}

Ce dossier, coordonné par Thierry Malbert et Gérard Pithon rassemble des articles de recherches menées dans la région du sud-ouest de l'océan Indien : Madagascar, Maurice, Mayotte, Réunion.

La famille, première instance de socialisation avant l'école, est sans aucun doute universelle. En effet, que la filiation soit biologique ou sociale (Fine, 2013), la famille, regroupant un certain nombre d'individus vivant sous un même toit, est partout présente sur la terre. Pourtant, l'organisation des relations sociales qui émergent de la structuration des réseaux de parenté varie considérablement d'une société à l'autre. Par exemple, la structure de parenté des Inuits du Groenland est différente de celle des Dogons du Mali ou encore de celle des Aborigènes d'Australie (Lévi-Strauss, 1967 ; Goody, 1985). Chaque culture modèle une organisation qui structure et identifie des réseaux de parenté. Les adultes peuvent y exercer des rôles parentaux en développant certaines compétences relationnelles en vue d'éduquer leur(s) enfant(s) (Pithon et Terrisse, 2015). Si les organisations familiales sont différentes et plurielles en fonction des cultures et de leurs évolutions, on constate cependant qu'elles ont toutes un point commun : socialiser l'enfant dans le but de le

\footnotetext{
${ }^{1}$ Thierry Malbert, ICARE, EA 7389 (Institut Coopératif Austral de Recherche en Éducation), Université de la Réunion, France.

Directeur scientifique de l'observatoire de la parentalité de La Réunion.

Président du CERVOI : Centre d'Enseignement et de Recherche sur les Valeurs dans l'Océan Indien. Coopération bilatérale Maurice-Réunion.

Contact : thierry.malbert@univ-reunion.fr

Gérard Pithon, EPSYLON, EA 4556 (Equipe Conflits, engagement, éthique, société), Université Paul Valéry, Montpellier, France.
}

Contact : gerard.pithon@gmail.com 
rendre autonome et de lui permettre d'intégrer la société, en respectant ses normes culturelles.

La diversité culturelle de la partie occidentale de l'océan Indien est très forte. Cette région offre des spécificités culturelles variées, certaines y sont ancestrales (Comores, Madagascar), d'autres beaucoup plus récentes (Maurice, Réunion, Seychelles) (Barat, 1990). Ces sociétés postesclavagistes ont des points communs : 1) avoir surmonté en partie les traumas de l'esclavage avec ses conséquences dramatiques pour les identités personnelles, sociales (familiales et communautaires) et l'éducation des enfants; 2) avoir donné naissance à des identités culturelles spécifiques nommées sociétés créoles (Chérubini, 1994), suite à un brassage forcé de diverses populations.

L'océan Indien est un carrefour de civilisations, matérialisé par l'existence de «civilisations de frange », selon l'expression de l'anthropologue Ottino (1974), qui se sont développées dans différents archipels et îles. Pratt (1991) les qualifient aussi de civilisations issues de «zones de contact». Ces interfaces sont marquées à la fois par un continuum culturel mais également par des spécificités locales, ellesmêmes renforcées par l'insularité.) Depuis 5000 ans, l'océan Indien a fait l'objet d'innombrables navigations et d'échanges de toutes sortes (Sherer, 1994). Les cultures en contact qui s'y sont développées n'ont pas toujours eu des relations égalitaires entre elles (Fuma, 1998, 2002).

Les structures de parenté et les organisations sociales des familles des pays de l'océan Indien sont aussi diverses que leurs appartenances culturelles, elles-mêmes émanant de la richesse de leurs rencontres (Poirier, 1999 ; Malbert, 2015). D'une appartenance filiale majoritairement patrilinéaire à Madagascar à une matrilinéarité renforcée aux Comores (Blanchy, 1990), c'est dans les sociétés créoles réunionnaises, mauriciennes et seychelloises que la diversité des systèmes de parenté en contact, unilinéaire ou bilinéaire, est la plus présente. Ainsi cette diversité culturelle intrafamiliale apparaît lors des cérémonies de mariage ou lors des rituels en lien avec l'éducation de l'enfant. Par exemple, l'importance donnée aux «cheveux maillés », autrement dit, l'emmêlement de la chevelure des nourrissons à La Réunion, est parfois perçu par les parents comme un signe de l'emprise maléfique d'un esprit, ancêtre ou divinité, issu aussi bien de la filiation malgache que de la filiation indienne. Le rituel thérapeutique institué pour amener un certain apaisement, dans les champs du visible et du non-visible, contient à la fois une base commune aux deux filiations mais repose en outre sur une ritualisation catholique devant une statue de la vierge Marie (DumasChampion, 1993). Cet exemple permet d'analyser les représentations 
croisées présentes dans les sociétés où les métissages biologiques et culturels sont extrêmement forts et structurés.

La question de la diversité est devenue en ce début de millénaire un enjeu majeur dans un grand nombre de sociétés. Cette thématique est désormais omniprésente dans les sciences humaines et sociales. $\mathrm{La}$ Déclaration universelle de l'Unesco sur la diversité culturelle (UNESCO, 2001) est un instrument normatif reconnaissant pour la première fois cette diversité comme un " héritage commun de l'humanité » et un impératif éthique inséparable du respect de la dignité humaine.

Qu'en est-il de la diversité culturelle des modèles éducatifs intrafamiliaux ? Selon l'anthropologie de l'éducation (Wulf, 1999) l'éducation des enfants au sein des familles est fortement en lien avec les référents culturels, compris sous leurs formes acculturatives. Cette diversité, issue des périodes esclavagistes, coloniales et postcoloniales, puis du développement de la mondialisation et des problématiques migratoires, est aujourd'hui de plus en plus présente dans de nombreux espaces de la planète. Au sein des familles se pose alors la question du choix des invariants culturels et cultuels pour éduquer les enfants. Certains parents font le choix commun d'une éducation mono-culturelle exclusive à un seul lignage, d'autres incitent l'enfant à respecter à la fois les normes culturelles et cultuelles issues des lignées maternelle et paternelle. Dans ce cas précis les deux cultures parentales sont alors sollicitées dans un cadre éducatif pluriculturel. Pour d'autres parents, les choix éducatifs sont beaucoup plus larges, ils dépassent la logique des héritages liés à la filiation, certains enfants eux-mêmes vont valoriser ou non certaines affiliations, y compris différemment selon les âges et les contextes. Faire vivre les traditions ou s'approprier des références culturelles tout en abordant la modernité et ses évolutions est désormais pour les parents un défi, voire un conflit à surmonter. Il s'agit en effet de trouver un équilibre suffisant afin de continuer à transmettre des valeurs ou renouer avec des références du passé, en accord avec celles du présent et pour préparer l'avenir (Debray, 1997).

La transmission d'une culture, dans et par la famille, a des conséquences importantes sur l'éducation et la socialisation primaire des enfants (Georgas, Berry, Van de Vijver, Kagitçibasi et Poortinga, 2006 ; Malbert et Latchoumanin, 2007). Dans les sociétés multiculturelles, où de nombreuses religions cohabitent, comme c'est le cas dans les îles de la région du sud-ouest de l'océan Indien (Réunion, Madagascar, Mayotte, île Maurice...), la question de l'«arbitrage » entre les valeurs de diverses communautés culturelles et religieuses en présence (chrétiennes, musulmanes, animistes, hindoues...) ne se pose pas nécessairement 
(Simonin et Wolff, 2003 ; Malbert, 2010a). Un continuum biologique et culturel ancien, ayant un ancrage sur plusieurs générations, permet à l'individu de reconnaitre en lui ses multi-appartenances et héritages (Bonniol, 1988) et ce mouvement est accentué par le besoin et le devoir de mémoires. Au cœur des sociétés créoles, on peut adopter la proposition de Lahire (1998) « le singulier est nécessairement pluriel ».

Á La Réunion, la présence d'un groupe de dialogue inter-religieux actif est reconnue par la population comme vecteur de convergence et de cohésion sociale (Malbert et Rafidinarivo, 2015). Cette logique de rencontre plurielle, suscitant un dialogue ocuménique pacifique dans le respect de la diversité, a un impact positif chez les parents dans la gestion des modèles éducatifs transmis aux enfants et dans celle des conflits interculturels intrafamiliaux. La coexistence pacifique entre les différents cultes présents sur le territoire de l'île de La Réunion, est un exemple qui pourrait venir éclairer et stimuler d'autres analyses contribuant à développer des stratégies sociales du « vivre ensemble » (Malbert, 2013; Cambefort, 2001) dans des territoires en tension. Bien que certains auteurs soutiennent que la coexistence pacifique des religions est souvent un mythe (Luffin et Weis, 2013), l'expression de la laïcité vécue dans ce département français d'outre-mer, permet à la pluralité des valeurs familiales de coexister dans le respect et la dignité de leur singularité.

L'objectif de ce dossier de la Revue internationale de l'éducation familiale est de mieux comprendre, au-delà de la place de la culture d'origine et des processus d'acculturation (Belhandouz, 2005), comment s'élaborent et se transmettent les valeurs culturelles dans les familles (Schwartz et Bilsky, 1987), tout particulièrement pour celles des sociétés de l'océan Indien (Deschamps, 2009 ; Malbert, 2010c ; Rizzo et Malbert, 2011).

L'archipel des Comores constitué de quatre îles situées dans le canal du Mozambique - Grande Comores, Anjouan, Mohéli et Mayotte - est l'espace le plus anciennement peuplé des îles de l'océan Indien (Barraux, 2009). Plus proche géographiquement du continent africain, les îles de la lune (Damir, Boulinier et Ottino, 1985), ont été peuplées à partir du début du premier millénaire par des populations austronésiennes, puis par des populations musulmanes arabo-perses, originaires du Yémen et d'Iran. Des sultanats vont être mis en place à partir du XIII ${ }^{\text {ème }}$ siècle et des populations Africaines Bantous sont alors déportées et soumises à l'esclavage. L'île de Mayotte, faiblement peuplée et n'ayant aucune oligarchie fut longtemps dominée par le sultan de l'île d'Anjouan et par les royautés Malgaches (Kana-Hazi, 1996). L'arrivée des européens au $\mathrm{XVII}^{\text {ème }}$ siècle va progressivement changer les relations entre les îles. Le 
traité de session de Mayotte à la France en 1841 va permettre à cette île, notamment lorsque l'ensemble de l'archipel deviendra un protectorat en 1885, puis une colonie française en 1912, de devenir progressivement la capitale de la colonie des Comores (Aïnouddine et Charpentier, 2012).

Figure 1 : Localisation des Comores, de Mayotte, de Madagascar, de La Réunion et de Maurice

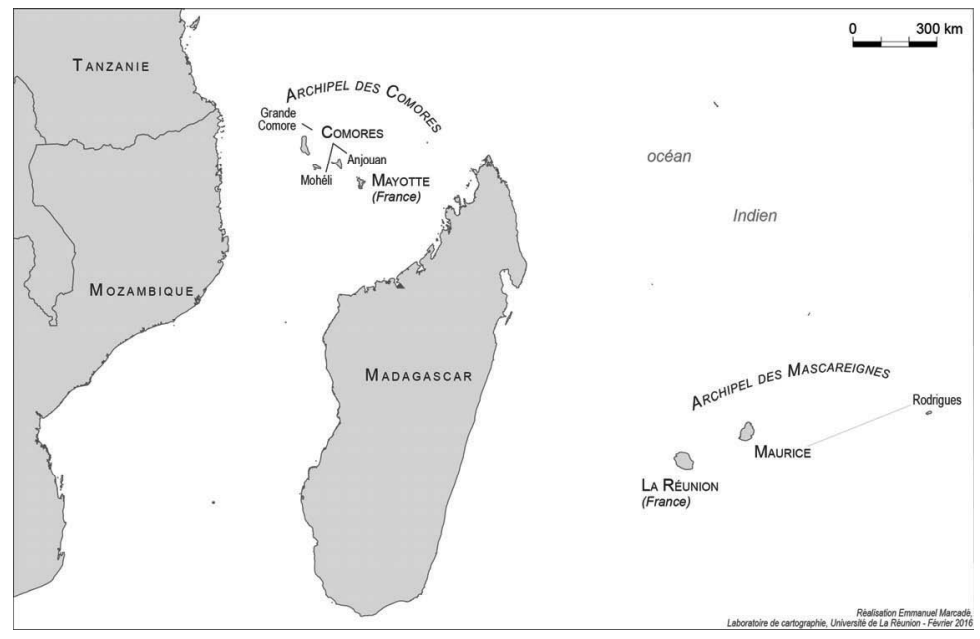

Laboratoire de cartographie, Université de La Réunion, fevrier 2016

En 1975 la population de Mayotte souhaitant garder cette suprématie face aux trois autres îles de l'archipel, choisit de rester sous le giron de la France en refusant l'indépendance (Fontaine, 1995). Alors que le reste de l'archipel est géré par une République Islamique, puis par l'Union des Comores, depuis cette date la volonté politique des mahorais est cependant d'affirmer plus encore leur attachement à la République française. Passant du statut de collectivité départementale en 2000 à celui de département d'outre-mer en 2011, Mayotte devient, suite à un référendum approuvant cette volonté à plus de $95 \%$, le $101^{\text {ème }}$ département français (Bertile, 2012). L'ensemble des populations de l'archipel des Comores, issues de divers contacts et rencontres ethnoculturelles - apports austronésiens, bantous, arabes et plus tardivement européens - ont néanmoins des langues propres à chaque île. 
C'est dans le cadre du rapport à la pluralité langagière à Mayotte que s'inscrit l'article de Foued Laroussi (Université de Rouen) intitulé La transmission des langues et cultures à Mayotte : quels enjeux identitaires pour la famille et l'école? L'auteur, dont certaines publications portent sur les langues et leur contexte (Laroussi, 2007, 2010), présente les résultats d'enquêtes sur la transmission actuelle des langues dans cette île, parmi lesquelles le français. Cette transmission apparait comme un enjeu identitaire pour la famille et l'école. Selon cet auteur, la transmission des langues au sein de la famille et leur enseignement à l'école n'obéissent pas à la même logique. Le français, autrefois exclu de la sphère familiale, commence à y être présent conformément aux attentes des jeunes. Par contre pour la génération des plus de quarante ans, le shimaore et le kibushi (langues locales) restent les principales langues utilisées au sein de la famille mahoraise. Comment les Mahorais perçoivent-ils cette situation ? Pourquoi la demande de reconnaissance et de prise en compte des langues premières à l'école s'accompagne-t-elle d'une forte revendication du français à l'école ? L'article apporte des éléments de réponses qui ne manquent pas de souligner le rapport étroit entre les valeurs traditionnelles empruntées à la religion musulmane et les valeurs plus modernes empruntées à la République française laïque. D'autres études ont montré que cette problématique soulève plus globalement la question des relations entre familles et écoles à Mayotte, et notamment entre tradition musulmane et laïcité (Malbert et M'trengoueni, 2016). Ces interrogations permettent de mettre à jour, dans le cas d'une évolution sociétale extrêmement rapide, les enjeux identitaires qui se greffent autour des conflits intergénérationnels et des ruptures dans la transmission (Malbert, 2010b). Le rapport à la laïcité à Mayotte est totalement nouveau. Les familles mahoraises, à 95\% musulmane, s'investissent progressivement, et parfois avec de nombreuses incompréhensions, dans cette nouvelle réalité éducative auprès de leurs enfants. Les écarts socioculturels entre l'ancien code civil des mahorais, le Minhâj-al-Tâlibîn traité de jurisprudence élaboré en Syrie au XIII ${ }^{\text {ème }}$ siècle (droit local), et le code civil de la République française (droit commun) sont en effet importants. La réinterprétation des faits éducatifs vécus et perçus dans une symbolique opérative d'une modernité laïque relative, semble se faire plus facilement chez les jeunes parents diplômés.

Le deuxième article de ce dossier concerne Madagascar. Essentiellement peuplée par des populations originaires d'Indonésie et d'Afrique australe, l'île connait très tôt un découpage territorial en dixhuit tribus hiérarchisées par une nomenclature à la fois géographique et ethnique et réparties entre les populations côtières et les populations des hauts plateaux. Les populations composées d'agriculteurs, d'éleveurs, de 
pêcheurs et de petits commerçants ont été pendant de nombreux siècles gouvernées par une royauté multiséculaire (Barrier, 1996). La grande île passera sous le statut de colonie française de 1897 à 1960 (Deschamps, 1972). Á partir de son indépendance ce pays va connaitre trois républiques successives. En 2008, une crise politique, économique et sociale sans précédent va plonger le pays dans une très grande pauvreté. Ce contexte de difficulté accrue à tous les niveaux de l'échelle socioéducative est étudié par Gil Dany Randriamasitiana (Université d'Antananarivo, Madagascar), dans un article intitulé Cultures familiales et scolaires : réalités locales de familles défavorisées à Madagascar. Le texte est consacré aux familles en situation de grande précarité dans un quartier de la commune rurale de Tanjombato située au centre de Madagascar. L'auteur cerne les conditions d'apprentissages formels et informels des enfants dans des familles défavorisées des écoles de ce quartier, notamment quand la transmission des valeurs ancestrales et du patrimoine culturel est grandement compromise. Son approche sociologique de ce contexte de vie difficile des familles et des enseignants dans les écoles permet de comprendre le poids des déterminants socioéconomiques et sociocognitifs sur les apprentissages des enfants dans l'enseignement primaire.

Á Madagascar les valeurs sociales étaient autrefois enracinées dans l'ancestralité, l'appartenance clanique ou familiale (Ottino, 1998). Ainsi la référence à la terre des ancêtres et à une vision égalitaire était souvent présente dans la plupart des activités socioculturelles. De nos jours, les processus de transmission dans les familles des pratiques socioculturelles et des repères axiologiques ancestraux, encore en vigueur, sont difficiles à perpétuer dans des situations de pauvreté extrême. L'analyse présentée permet d'étayer les réflexions sur les effets de la précarité comme obstacle à la transmission des valeurs sociales et éducatives. Le contexte de fragilité de certaines familles malgaches ne fait-il pas là écho à bon nombre de situations que peuvent vivre actuellement dans le monde des parents issus de migrations contraintes ? Cet article ouvre une piste de réflexion sur les liens entre la crise de la transmission signalée par Singly de (2003) et son accentuation lorsque la précarisation ne permet plus de références aux traditions éducatives.

La situation de l'île de La Réunion est tout à fait différente. Cette île a été peuplée à partir de 1663 successivement par divers apports de populations venues de leur plein gré - aventuriers, petits planteurs, gros colons, commerçants - ou de force, comme ce fut le cas pour les esclaves et certains engagés. Les réunionnais sont en lien avec de multiples berceaux, d'origines malgache, européenne, africaine, indienne 
musulmane, indienne tamoul, comorienne, chinoise (Fuma, 1994, 2002). Les groupes ethnoculturels en présence se sont vu assigner dès le départ des positions économiques et des statuts fort inégaux. En effet cette société polyethnique fut longtemps hautement hiérarchisée sous le contrôle d'une métropole européenne - la France - et d'une aristocratie locale qui la représentait (Benoist, 1991). L'abolition de l'esclavage en 1848 , a donné lieu à de nouveaux déplacements d'hommes et de femmes engagés pour le travail dans les plantations. L'accès au statut de département d'outre-mer français en 1946 a permis successivement deux phases de décentralisation donnant progressivement un peu plus d'égalité et de liberté à l'ensemble de sa population.

La diversité des rencontres biologiques et culturelles, souvent issue d'un fort déséquilibre entre les sexes (plus d'hommes que de femmes dans chaque communauté entrante) a progressivement donné naissance à une société créole fortement métissée, dans laquelle l'hérédité, dans sa dimension anthropologique et sociale, apparaît comme un des enjeux de l'identité (Malbert, 2010a). Á la Réunion, la forte diversité des origines géographiques de la population (Afrique, Madagascar, Comores, Europe, Inde et Chine), offre à travers le métissage, un panel phénotypique très diversifié au sein de la plupart des familles. Le résultat des différentes rencontres permet de concevoir des représentations singulières touchant ce qu'il est possible d'appeler l'hérédité : la transmission au fil des générations de caractères distinctifs. Ces traits constitutifs de la personne ou du groupe, qu'ils soient liés à l'hérédité biologique et/ou culturelle sont à la base de la construction identitaire individuelle et familiale. Peut-on faire l'hypothèse, comme Benoist et Bonniol (1991), que l'identité sociale et culturelle d'une famille (résultat de l'entrecroisement des patrimoines héréditaires) n'est pas le résultat de toute la descendance mais le résultat d'une stratégie sociale de l'hérédité ? D'autres études portant sur cette question (Gleize, 1994; Malbert, 2010a), montrent comment les représentations de l'hérédité apportent une compréhension plus fine de la structure de la parenté, de l'identité et des relations interculturelles. La représentation des discours sur les ressemblances familiales - physiques et/ou morales - entre affiliés, structurent au sein de la parenté des appropriations symboliques des enfants entre les lignées maternelles et/ou paternelles. En société créole, cette symbolique a une forte résonnance sur la responsabilité éducative des parents notamment à propos des projections qu'ils peuvent faire sur le devenir de tel ou de tel autre enfant.

C'est sous le rapport de la pluralité des héritages biologiques, culturels et cultuels, et de leur complexité propre à l'histoire de la société créole réunionnaise, que Gérard Pithon, (Université Paul Valéry, 
Montpellier, France) et Randjani Ponin Collège Saint Michel ; Chambre de Commerce et d'Industrie à Saint-Denis de La Réunion), proposent un article intitulé Transmission des valeurs à La Réunion sur trois générations d'hommes ou de femmes, catholiques ou hindoues.

Comment s'opère à La Réunion la transmission des valeurs entre plusieurs générations appartenant à des communautés religieuses différentes? Á partir des modèles théoriques définissant les valeurs et leur catégorisation présentée par Schwartz (1992), Pithon et Ponin étudient les similitudes ou les différences entre les systèmes de valeurs liés au genre, aux générations, par des membres appartenant à l'une de ces deux religions et aux proximités éventuelles des valeurs accentuées par les liens de famille au sein des filiations. Les résultats font apparaitre des données intéressantes à propos de valeurs telles que l'individualisme, l'hédonisme, la bienveillance, l'autonomie, la sécurité, la conformité, la tradition, l'universalisme, la stimulation, la réussite et le pouvoir, qui se transmettent différemment en fonction des filiations hindoues ou catholiques et en fonction des générations. Les valeurs sont une des dimensions éducatives intéressantes à étudier pour cerner des tendances qui apparaissent au fil du temps sur le rapprochement ou la distanciation entre certaines communautés cultuelles. $\mathrm{Si}$ au $\mathrm{XXI}^{\text {ème }}$ siècle certains conflits interreligieux semblent s'étendre, la Réunion connait une cohabitation pacifique entre les communautés cultuelles en présence sur son sol. Si cette réalité peut être adoptée comme référence à étendre audelà de l'océan Indien, il est important de signaler toutefois que cette reconnaissance de la diversité cultuelle est récente. Jusqu'au début des années 1960, les pratiques culturelles et cultuelles autres que chrétiennes étaient assez discrètes. La hiérarchisation culturelle issue du monde colonial et de la domination occidentale se traduisait dans les représentations sociales hiérarchisées des religions et dans leur traitement administratif. La politique territoriale française considérait ce type d'unicité culturelle comme le garant et le fondement de la légitimité territoriale ainsi que de la cohésion nationale, en outremer plus qu'ailleurs, en raison des spécificités de son peuplement sur des territoires éloignés. Á partir des années 1980 la donne a progressivement changé, La Réunion se caractérisant par un dialogue entre des cultes, progressivement devenu garant de la cohésion sociale.

Ces dynamiques interreligieuses, qui interpellent, agissent sur la cohésion sociale (Malbert et Rafidinarivo, 2015). L'approche contextuelle de la réalité réunionnaise et l'émergence de dialogues interreligieux nous paraissent nécessaire pour bien comprendre les dynamiques cohésives qui vont susciter l'action du Groupe de Dialogue Interreligieux de la Réunion, 
association crée à La Réunion dans le but de développer l'interculturalité, l'inter-religiosité et la socialisation cohésive (GDIR, 2005). La contribution du GDIR dans l'espace public réunionnais influe sur les dynamiques de la cohésion sociale : sa posture politique pour la paix, sa participation politique citoyenne et sa contribution au " vivre ensemble » permettent d'activer des valeurs de respect, de partage et de paix, de véhiculer une sérénité vécue et ressentie par la population (Malbert et Rafidinarivo, 2015). Dans ce cadre, les recherches sur la transmission ou la non-transmission des valeurs dans la filiation, adossées à la variable religion, ont toute leur importance pour mieux comprendre et analyser les évolutions des liens intercommunautaires.

L'île Maurice, société créole par excellence, peuplée à partir du $\mathrm{XVI}^{\text {ème }}$ siècle par des européens, des africains, des indiens (hindous ou musulmans) et des chinois, fut sous domination hollandaise (1598-1710), puis française jusqu'en 1810 et finalement anglaise jusqu'à son indépendance en 1968. Bien que liée, comme La Réunion, à des logiques globalisantes assimilatrices, c'est dans le contexte du multi-culturalisme et moins dans celui de l'inter-culturalisme (Malbert, 2013), que les logiques de rencontres intercommunautaires se sont mises en place depuis plusieurs décennies. Contexte fragile lié aux espaces îliens dépendants des grands pôles économiques continentaux, la prise en compte de la diversité culturelle et cultuelle à l'île Maurice est récente (Malbert et Latchoumanin, 2007). Si par le passé des conflits inter-ethniques (Asgarally, 2005) ont pu marquer au fer rouge les appartenances identitaires, notamment entre créoles et hindous, depuis 2008 une évolution vers une reconnaissance de toutes les appartenances se construit peu à peu (Asgarally 2013). Une logique de rencontre intercommunautaire, interculturelle, s'inscrit désormais, tant dans le domaine universitaire, selon l'écrivain Jean-Marie Le Clézio (2009), que dans les contextes secondaire et primaire. L'île Maurice s'engage donc vers une reconnaissance et une valorisation de toutes les composantes de sa population.

L'éducation par et avec les valeurs (CERVOI : Centre de Recherche et d'Enseignement sur les Valeurs dans l'Océan Indien, 2015) se met en place dans les écoles. Les associations civiles, notamment à partir d'activités artistiques, interviennent de plus en plus dans les classes. C'est dans cette nouvelle dynamique coéducative qu'Emilie Carosin (ONG Tipa, île Maurice ;Université de Clermont-Ferrand, France), propose un texte sur Les défis de la valorisation personnelle d'adolescents mauriciens grâce à des formations artistiques. 
Cet article met en exergue les enjeux de la reconnaissance et de la valorisation des adolescents pour qu'ils se réalisent pleinement à partir de formations artistiques. La synthèse de dix études de cas, réalisées à partir de l'observation systématique des conduites d'adolescents de 13 à 15 ans en ateliers d'arts plastiques, et l'analyse de leurs discours sur leurs projets professionnels, montrent le désir des sujets d'être reconnus par leur participation aux ateliers. La contribution qu'ils souhaitent apporter à leur famille et à leur quartier par le biais de leur projet professionnel est envisagée en relation avec les compétences professionnelles qu'ils souhaitent développer. Ils aspirent à devenir des modèles pour leur entourage, et ce malgré les dépréciations auxquelles ils font face. $\mathrm{La}$ reconnaissance et la diversité passent aussi par la valorisation de l'individu apprenant.

Les apports scientifiques relatifs aux problématiques soulevées dans ce dossier permettront aux lectrices et aux lecteurs de mieux apprécier des réalités éducatives familiales appartenant à d'autres cultures, issues notamment d'îles de l'océan Indien, voire de mieux être en capacité de tisser des liens avec des personnes indocéaniennes.

Les questions abordées dans ce dossier sont, nous le verrons, complexes : langues parlées en famille et à l'école à Mayotte, influence de la précarité sur l'abandon des traditions entre une éducation informelle et formelle à Madagascar, transmission de valeurs familiales, entre ruptures et/ou continuités, selon les générations et les cultes à La Réunion, soutien des jeunes par les arts pour affirmer leur identité et faciliter leur insertion à l'île Maurice. Les articles de ce dossier concernent de surcroit la délicate question de la transmission des valeurs et de la culture dans des îles de l'ouest de l'océan Indien ayant connu l'esclavage et l'engagisme. Ces contextes éducatifs interculturels ne manqueront pas d'interroger les chercheurs et les praticiens en éducation familiale, notamment ceux qui accompagnent des parents vivant en contexte de grande pauvreté.

\section{Références bibliographiques}

Aïnouddine, S. et Charpentier, M. (2012). Études sur l'archipel des Comores, Rapport CNDRS. Moroni.

Asgarally, I. (2005). L'interculturel ou la guerre (Préface de J.M.G. Le Clézio). Maurice : Éditions M.S.M.Ltd.

Barat, C. (1990). Classification et typification dans un contexte multiculturel. Dans J.-F. Reverzy et J.-C. Marimoutou (dir.), L'espoir transculturel, tome 2, Iles et fables (p. 68-89). Paris : L'Harmattan. 
Barraux, R. (2009). Du corail au volcan : l'histoire des îles Comores. Moroni : Komedit.

Barrier, M-F. (1996). Ranavalo, dernières reine de Madagascar. Paris : Balland.

Belhandouz, H. (2005). Pour une approche anthropologique de l'éducation familiale. La question de la socialisation des filles maghrébines en France. Dans H. Desmet et J-P. Pourtois (dir.), Culture et bientraitance (p. 131-148). Bruxelles : De Boeck.

Benoist, J. (1991). Le métissage : biologie d'un fait social, sociologie d'un fait biologique. Dans Actes du colloque international métissages linguistique et anthropologique (p. 12-22). Saint-Denis de La Réunion, tome II.

Bertile, W. (2012). Mayotte à l'heure de la départementalisation. Paris : l'Harmattan.

Blanchy-Daurel, S. (1990). La vie quotidienne à Mayotte. Paris : L'Harmattan.

Bonniol, J.-L. (1989). Couleur et identités : le miroir des apparences dans la genèse des populations créoles. Thèse de Doctorat d'Etat. Université Aix-Marseille I, sous la direction de Philippe Joutard.

Cambefort, J.-P. (2001). Enfance et famille à La Réunion, une approche psychologique. Paris : L'Harmattan.

CERVOI. (2015). Centre d'Enseignement et de Recherche sur les Valeurs dans l'Océan Indien. Coopération bilatérale, Université de la Réunion/Mauritius Institut of Education. La supervision d'une expérimentation : l'école des valeurs pour professionnaliser. Paris : Rapport de la Fondation de France.

Cherubini B. (1996). La construction symbolique des identités dans le monde créole : exemples réunionnais. Dans A. Carénini, J.-P. Jardel et J. Poirier (dir.), De la tradition à la post-modernité (p. 267-281). Paris : PUF.

Damir, B., Boulinier, G. et Ottino, P. (1985). Tradition d'une lignée royale des Comores. Paris : L'Harmattan.

Debray, R. (1997). Transmettre. Paris : Odile Jacob.

Deschamps, H. (1972). Histoire de Madagascar, Mondes d'Outre-Mer. Paris : Berger-Levrault.

De Singly, F. (2003). Les uns et les autres. Quand l'individualisme crée du lien. Paris : Armand Colin.

Dumas-Champion, F. (1993). Les cheveux maillés ou le marquage de l'identité ethnique en milieu créole réunionnais, Nouvelle revue d'ethnopsychiatrie, 20, 105-118.

Dumas-Champion, F. (2008). Le mariage des cultures à l'île de La Réunion. Paris : Karthala. 
Fine, A. (2013). Parenté : liens de sang et liens de cœur. Dans V. Bedin et M. Fournier (dir.), La parenté en question (p. 40-51). Auxerre : Éd. Sciences Humaines et Sociales.

Fuma, S. (1994). Histoire d'un peuple, La Réunion (1848-1900). SaintAndré : Éditions Azalées.

Fuma, S. (1998). L'abolition de l'esclavage à La Réunion, Saint-André, G.R.A.H.T.E.R : Groupe de Recherche sur l'Archéologie et l'Histoire de la Terre Réunionnaise.

Fuma, S. (2002). Mémoire de l'esclavage dans les îles du sud-ouest de l'océan Indien : inventaire des fonds d'archives publiques et privées. Dans UNESCO, Regard sur l'Afrique et l'océan Indien. Paris : Le Publieur.

GDIR, Groupe de Dialogue Inter-Religieux de La Réunion (2000). La laïcité réunionnaise un visage de la laïcité française. Saint-Denis de La Réunion : Nouvelle Imprimerie Dyonisienne

Georgas, J., Berry, J. W., Van de Vijver, F., Kagitçibasi, Ç. et Poortinga, Y. (dir.) (2006). Families Across Cultures. A 30 Nation Psychological Study. Cambridge : Cambridge University Press.

Gleize, P. (1994). Théories savantes, conceptions communes actuelles de l'hérédité. Filiation et divergence : quelques exemples, Ethnologie française, 23(1),11-24. PUF.

Goody, J. (1985). L'évolution de la famille en Europe, préface de G. Duby. Paris : Armand Colin.

Kana-Hazi. (1997). Histoire des îles : Ha'ngazidja, Hi'ndzou'ani, Maïota et Mwali. Moroni : Djahazi Édition.

Lahire, B. (1998). L'Homme pluriel. Les ressorts de l'action. Paris: Nathan.

Laroussi, F. (2007). Mayotte une île plurilingue en mutation. Mayotte : Éditions du Baoboab.

Laroussi, F. (2010). Langues, identités et insularités : regards sur Mayotte. Rouen : PURH.

Le Clézio, J. M. G. (2012). L'interculturel, seul recours. Dans A. Geoffroy et $\mathrm{S}$. Geoffroy (dir.), Intégration/exclusion des minorités à la lumière de l'interculturalité, Alizés (Université de La Réunion), 36, 11-12.

Lévi-Strauss, C. (1967). Les structures élémentaires de la parenté. ParisLa Haye : Mouton.

Luffin, X. et Weis, M. (2013). Un regard critique sur les «mythes » de la coexistence interreligieuse, Cahiers de la Méditerranée, $n^{\circ} 86$.

Malbert, T. (2010a). Généalogie et représentation de l'hérédité dans les familles réunionnaises. Dans D. Le Gall et N. Roinsard (dir.), Chronique d'une autre France : la Réunion. Genre de vie et intimités créole (p. 221-239). Paris : L'Harmattan. 
Malbert, T. (2010b). L'identité à Mayotte : le nom de famille et sa réforme. Regard anthropologique. Dans M. Latchoumanin (dir.), Formation permanente et construction identitaires dans les îles de l'océan Indien (p. 173-187). Paris : Karthala.

Malbert, T. (2010c). Le rôle et la place de l'éducation interculturelle dans le développement durable des îles de l'océan Indien. Dans Cheikh Alâwi (dir.), Graines de lumière; héritages du Cheikh Alâwi: centenaire de la voie Soufi Alawiyya, 1909-2009 (p. 97-108). Beyrouth : Dar Albouraq.

Malbert, T. (2013). L'approche interculturelle de l'éducation au cœur des programmes scolaires. Dans I. Asgarally (dir.), Diversité culturelle et interculturelle : quelles assises pour la paix ? Fondation pour la Paix et l'Interculturalité, FIP. Préface de J.M.G. Le Clézio (p. 126136). Ile Maurice : Mahatma Gandhi Institut.

Malbert, T. (dir.) (2015). L'éducation familiale, quels nouveaux défis ? Parent, enfant et école. Paris : Karthala.

Malbert, T. et Latchoumanin, M. (2007). Famille et parentalité : rôles et fonctions entre tradition et modernité. Paris : L'Harmattan.

Malbert, T. et M'Trengoueni, M. (2016). Famille et écoles à Mayotte : entre tradition musulmane et rapport à la laïcité. Á paraître dans les actes du XVI ème congrès international de l'AIFREF (Bilbao), Symposium L'éducation à la citoyenneté et à la laïcité en famille et à l'école : enjeux et difficultés sous la direction de G. Pithon.

Malbert, T. et Rafidinarivo, C. (2015). Dialogue interreligieux et cohésion sociale à La Réunion. Dans L. Faberon (dir.), Liberté religieuse et cohésion sociale (p. 285-377). Aix en Provence : PUAM.

Malbert, T. et Rizzo, R. (2011). Le rapport du jeune collégien et de sa famille à l'école : le cas de La Réunion. Dans Y-S. Live et J-F. Hamon (dir.), Kabaro: Construction identitaire et interculturalité dans le monde Indo-océanique (p. 313-332). Paris : L'Harmattan.

Ottino, P. (1974). L'océan Indien comme domaine de recherche, L'Homme, Revue française d'anthropologie, 14(3-4), 143-151.

Ottino, P. (1998). Les champs de l'ancestralité à Madagascar. Parenté, alliance et patrimoine. Paris : Karthala.

Pithon, G. et Terrisse, B. (2015). Intervention précoce, parentalité, bientraitance et multiculturalité : enjeux et limites. Dans J.P. Pourtois et P. Kiprianos (dir.), Famille, École, Sociétés locales : Politiques et Pratiques pour l'Enfance (p. 132-144). UMONS : Éd. Éducation et Famille.

Poirier, J. (1999). Culture créole, hétéroculture réunionnaise. Dans C. Chérubini (dir.), La recherche anthropologique à La Réunion (p. 125-145). Paris : l'Harmattan. 
Pratt, M-L. (1991). Arts of the Contact Zone, Archived at University of Idaho, English 506, Rhetoric and Composition : History, Theory, and Research (p. 33-40). Profession 2006. New-York : MLA.

Scherer, A. (1994). La Réunion. Paris : PUF, 4e éd.

Schwartz, S. H. (1992). Universal in the content and structure of values : theoretical advances and empirical testes in 20 countries. Advances in Experimental Social Psychology. 25, 1-65.

Schwartz, S.H. et Bilsky, W. (1987). Toward a psychological structure of human values. Journal of Personality and Social Psychology, 53, 550-562.

Simonin, J. et Wolff, E. (2003). Familles et École à la Réunion. Regards anthropologiques et sociologiques. Dans F. Tupin (dir.), Univers créole, 3. (p. 143-168). Paris : Anthropos.

Wulf, C. (1999). Anthropologie de l'éducation. Paris : L'Harmattan. 